\title{
Metáforas ontológicas en el quechua ayacuchano: personificación y cosificación
}

\author{
IsABEL GÁlVEz Astorayme \\ Universidad Nacional Mayor de San Marcos \\ belisag@yahoo.com \\ ISABEL JUdith GÁLVEZ GÁlVEZ \\ Universidad Nacional Mayor de San Marcos \\ juditagg@hotmail.com
}

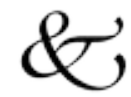

Resumen

El presente artículo describe y explica expresiones linguísticas correspondientes al entorno cultural de los quechuahablantes del dialecto ayacuchano. Estas expresiones manifiestan procesos cognitivos como la personificación y la cosificación. Nuestro estudio evidencia la manera en que los quechuahablantes conceptualizan su realidad inmediata en virtud del acercamiento que con ella experimentan. De este modo, la evidencia linguística respecto del discurso quechua permite sostener que existe un trazo invariable entre lengua y cultura en la mentalidad andina.

Palabras claves: metáfora quechua, metáfora conceptual, expresión metafórica

\begin{abstract}
This research describes and explains linguistic expressions for the cultural environment of Quechua speakers of the Ayacuchano dialect. These expressions manifest cognitive processes such as personification and objectification. Our study has revealed how the Quechua speakers conceptualize their immediate reality through the rapprochement under whichthey experience with. Thus, the evidence regarding the Quechua speech language allows to sustain anunchangedstroke between language and culture in the Andean mentality.

Keywords: Quechua metaphor, conceptual metaphor, metaphor expression
\end{abstract}




\section{Introducción}

Nuestro artículo enfoca un aspecto de la investigación«Metáforas en el quechua ayacuchano: un estudio semántico-cognitivo», que desarrollamos durante el año 2012 a través del Instituto de Investigaciones Linguísticas (INVEL). Este artículo tiene la finalidad de mostrar aspectos semánticos no abordados en los aportes de las investigaciones quechuas tradicionales (Parker 1965, Calvo 1993, Soto 2012, entre otros).

Analizamos expresiones del discurso quechua de los hablantes de las zonas de los departamentos de Ayacucho, Huancavelica y parte occidental de Apurímac, zonas correspondientes al área dialectal Q.II -C (Cf. Torero 1964, 2002). Estas expresiones permiten conocer la manera en que los quechuahablantes del referido dialecto conceptualizan parte de su entorno inmediato a partir de la experiencia de la vida cotidiana.

Las expresiones materia de análisis del presente artículo contienen mecanismos cognitivos de naturaleza ontológica, aquellos que están referidos a los procesos de personificación y cosificación. Estos mecanismos parecen facilitar la concepción de determinadas entidades de alta complejidad en la cosmovisión andina.

Para el estudio y análisis de las metáforas en el discurso quechua ayacuchano recurrimos al marco teórico de la linguística cognitiva (Cf. §. 1). En este marco describimos y explicamos la semántica de las metáforas ontológicas (Cf. $\S .2$ ), concretamente aquellas expresiones en las que intervienen procesos cognitivos como la personificación (Cf. §. 2.1.) y la cosificación (Cf. §. 2.2.). Finalmente, presentamos las conclusiones que derivan de nuestras observaciones respecto del habla y pensamiento quechua (Cf. §. 2.3.).

\section{Metáforas en el seno de la lingüística cognitiva}

Los estudios tradicionales acostumbran definir las metáforas como mecanismos que se emplean únicamente con fines retóricos y poéticos (u ornamentales). Este modo de abordarlas cambió con la llegada del paradigma conocido como linguística cognitiva, a partir de la publicación de la célebre obra de Lakoff y Johnson MetaphorWe Live By (1980). El aporte medular de esta obra reside en la reformulación de la visión tradicional de la metáfora, pues en la nueva propuesta se asume que aquellas forman parte de nuestro conocimiento, de cómo las personas hablamos acerca de conceptos abstractos a partir de otros más básicos y la manera en que dichos conceptos nos permiten organizar, comprender y vivir el mundo.En todo análisis cognitivo de las metáforas es importante tener en cuenta la diferencia que existe entre metáforas conceptuales y expresiones metafóricas; las primeras se entienden como aquellos mecanismos 
que conceden un valor conceptual a las expresiones, mientras que las segundas forman parte de la evidencia empírica respecto de cómo los hablantes conceptualizan su entorno inmediato. Esta distinción "permite desvelar generalizaciones que, de otro modo, quedarían ocultas”(Cuenca y Hilferty 1999: 100).

Se presenta, a continuación, el esquema formal de la metáfora:

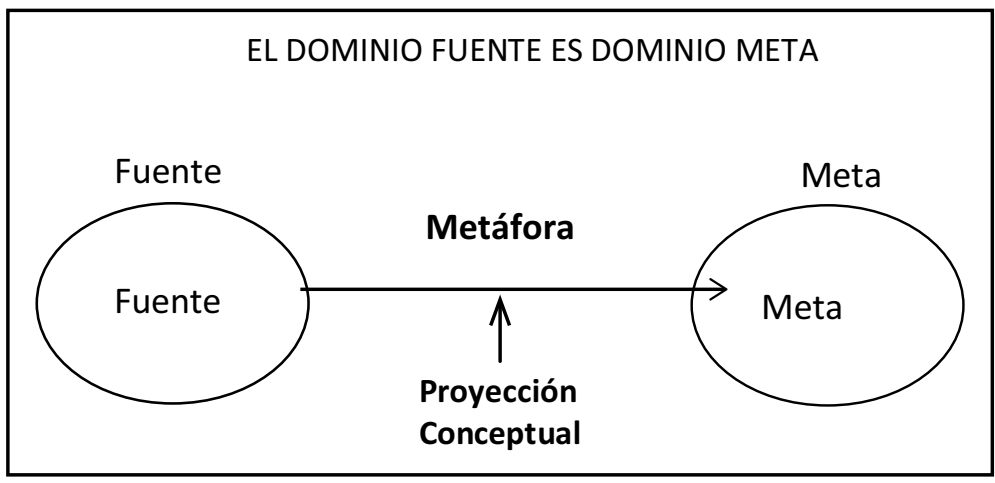

Fig. 1 Caracterización de la metáfora en la teoría de la metáfora cognitiva (TMC) (Lakoff y Jhonson 1980)

Las metáforas conceptuales se clasifican del modo siguiente: metáforas ontológicas, metáforas estructurales y metáforas orientacionales.

Las metáforas ontológicas determinan la concretización de entidades inmateriales, con lo cual se proyecta un concepto con características concretas hacia otro concepto carente de aquellas. Se distinguen tres tipos de metáforas ontológicas: aquellas en las que las entidades carentes de existencia física adquieren atributos propios de las personas (personificación); aquellas en las que la materialización supone la atribución de ciertos rasgos propios de las cosas (cosificación); y aquellas en las que las referidas entidades adoptan atributos concretos no de personas ni cosas, sino de plantas o animales (animicidad).

\section{Semántica de las metáforas ontológicas en el quechua ayacu- chano}

En este apartado detallamos dos maneras en que a partir de cierta evidencia linguiísticaes posible mostrar la forma en que el hombre andino conceptualiza las entidades inmateriales e inanimadas con las que mantiene una relación a través de un marco experiencial directo: la personificación yla cosificación. 


\subsection{Personificación}

Las expresiones que se circunscriben en esta clase de metáforas evidencian un proceso de personificación en dos direcciones: en la primera, el proceso supone la vitalización total de algunas entidades; la segunda se caracteriza por la extensión de una cualidad particular.

\section{(A) LAS ENTIDADES DE LA NATURALEZA SON PERSONAS}

Chay runam yakuta chakraman pusanqa.

Sig. Lit.: «Esa persona agua hacia chacra guiará».

Sig. Fig.: Esa persona llevará el agua hacia la chacra.

En la expresión anterior notamos que el nombre yaku 'agua' es pasible de guía a través del verbo pusa-y 'conducir'. Esto es, el agua como elemento natural puede ser guiada por una persona como si se tratara de una entidad con vida; por ello se usa el verbo en cuestión con la finalidad de resignificar tal realidad por medio de la personificación. La representación del caso se esquematiza del modo siguiente:

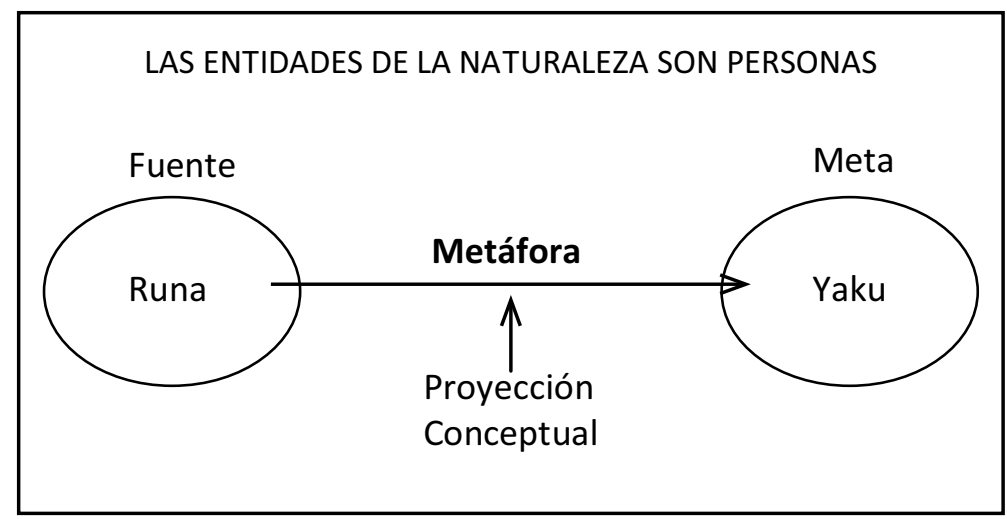

Fig. 2. Personificación de yaku 'agua'

(B) LAS DEBILIDADES SON OPONENTES

Wak runata qilla kaynin atiparqun.

Sig. Lit. : "A ese hombre su flojera venció».

Sig. Fig.: Ese hombre tuvo flojera.

Esta oración pone de manifiesto cómo determinadas entidades son concebidas como personas. Observamos que una entidad abstracta inexistente como 
el concepto qilla kay 'flojera' es conceptualizada -en el contexto cultural andino- como si se tratase de una persona capaz de oponerse a otra. Esta visión particular se constata en el sistema quechua por el empleo del verbo atipa $-y$ 'vencer', el cual supone un conflicto entre dos entidades animadas. En suma, el referido concepto inmaterial adquiere existencia física a través de un proceso de personificación.

\section{(C) LOS ESTADOS DE REPOSO SON OPONENTES}

Warmachata puñuy atiparqun.

Sig. Lit. : «A niñito venció sueño».

Sig. Fig. : Al niño le venció el sueño.

El enunciado que mostramos manifiesta que los estados de reposo son también conceptualizados como oponentes. De este modo notamos que la misma metáfora se articula para conceptos diferentes. Este esquema se explica en la cosmovisión andina quechua -nuevamente-por la presencia del verbo atipa $-y$ 'vencer'.

\section{(D) LOS ANIMALES SON PERSONAS}

Punta gallu waqayta hatarisunchik.

Sig. Lit.: «Primer gallo llorar levantaremos».

Sig. Fig.: Al primer canto del gallo nos levantaremos.

La expresión anterior muestra la dirección diferente que sigue el proceso de personificación en la mentalidad andina. El qallu 'gallo' no es una entidad carente de existencia física, pero el proceso de personificación es ciertamente aún persistente en la cultura quechua. En este caso se observa que el referido animal es mentalizado por el quechuahablante como si fuera una persona; la comprobación de lo dicho se evidencia en el empleo del verbo waqay 'llorar', lo que es característico de los seres humanos, pero que en este casoes atribuido al ave. El siguiente esquema grafica el proceso descrito: 


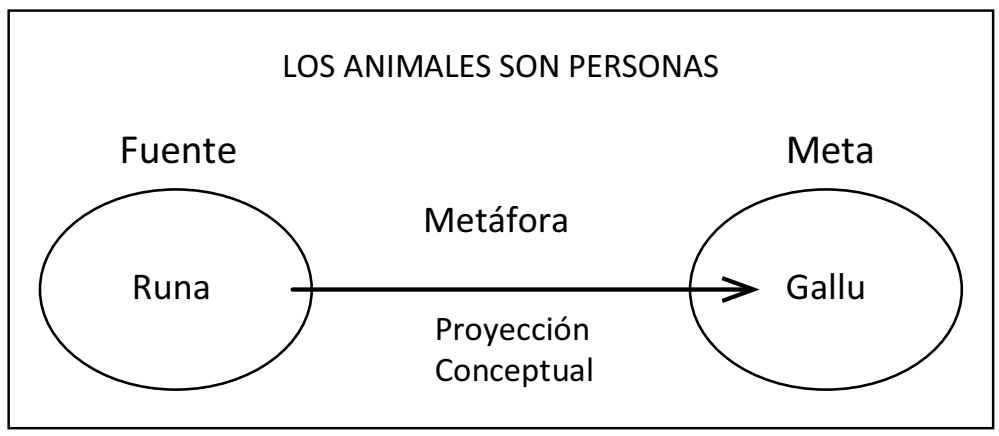

Fig. 3. Personificación del gallu

\subsection{Cosificación}

Las expresiones que están comprendidas dentro del proceso de cosificación manifiestan una variedad enorme de esquemas conceptuales. Encontramos expresiones que se enmarcan dentro de los patrones siguientes: objetivización, localidad y dimensionalidad en las entidades inmateriales.

\subsubsection{Objetivización en las entidades inmateriales}

El conjunto de enunciados que exponemos a continuación identifica el modo peculiar en que el nativohablante quechua de la variedad ayacuchana otorga existencia física a aquellas entidades que adolecen de ella. En esta clase de expresiones se "objetiviza» a aquellas entidades carentes de existencia física.

\section{(A) LAS INFIDENCIAS SON OBJETOS \\ Kuwintu qipiq \\ Sig. Lit. : «Cuento cargador» \\ Sig. Fig. : Correveidile}

La comprensión del sentido figurado en la expresión del caso (kuwintu qipiq 'infidente o chismoso') se produce en virtud de que el hablante quechua ha interiorizado el concepto de kuwintu 'chisme' como si fuese una entidad concreta que pudiese cargarse y, en consecuencia, pasible de ser movida de un lugar a otro. El chismoso o infidentees aquella persona que en la cultura andina se caracteriza por llevar un cuento de lugar en lugar. La presencia del verbo qipi-y 'cargar' explica la particularidad de su uso en la cosmovisión andina quechua. 
Por ello postulamos la metáfora conceptual LAS INFIDENCIAS SON OBJETOS. El siguiente esquema ilustra el caso:

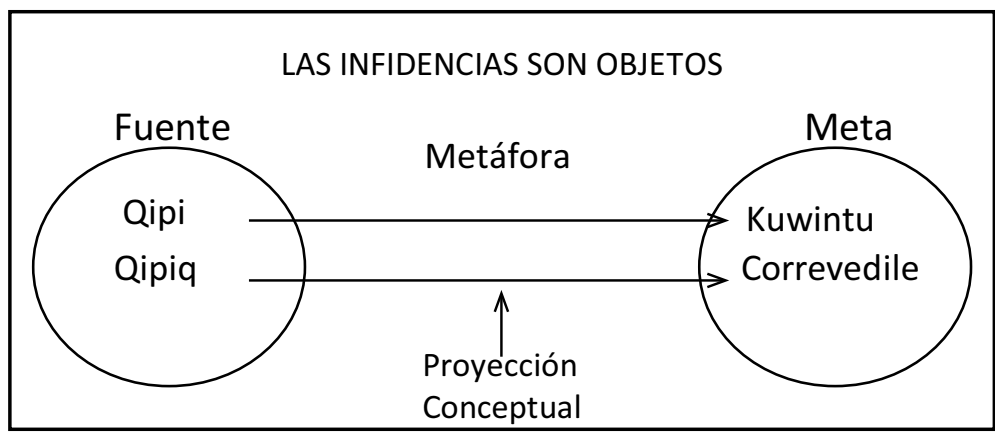

Fig. 4. Objetivización en las entidades inmateriales

\section{B) LAS FALTAS SON POSESIONES}

Nuqa mana huchayuq kani.

Sig. Lit.: «Yo no soy que tiene culpa».

Sig. Fig.: Yo no soy el culpable.

Estamos frente a un enunciado que revela que las entidades inmateriales se reanalizan a través de un aspecto muy propio de las entidades que no pertenecen al mundo de lo intangible: la cualidad de ser poseídas. En la cultura quechua, el huchayuq 'el que ha cometido un delito o que es responsable de aquel' se entiende como el posesor sobre quien la responsabilidad es deslindada. Este pensamiento está contenido en el morfema -yuq que deriva nombres a partir de otros nombres, sufijo que define un poseedor, en este caso huchayuq 'el que tiene la culpa'. En este sentido, proponemos la metáfora conceptual LAS FALTAS SON POSESIONES, pues ella caracteriza los matices que corresponden al universo cultural quechua.

En la metáfora siguiente, otro aspecto es reanalizado, a saber, la cuantificación.

\section{(C) LOS ESTADOS DE ÁNIMO SON CANTIDADES}

Achka kusisqam

Sig. Lit. : «Bastante alegre»

Sig. Fig. : Muy alegre 
El ejemplo ilustra que los estados de ánimo pueden ser reanalizados como objetos y, por tanto, posibles de ser contabilizados. El pensamiento quechua permite que en la manifestación del estado de ánimo kusi 'alegre' este se cuantifique por la presencia del cuantificador achka 'bastante' en la expresión, con lo cual el referido concepto es medible. Dicho ello, se plantea la metáfora conceptual siguiente: LOS ESTADOS DE ÁNIMO SON CANTIDADES.

D) EL AMOR ES UNA UNIDAD

Warmiywan rakinakurquniñan.

Sig. Lit. : «Con mi mujer ya me separé».

Sig. Fig. : He terminado mi relación con mi mujer.

La expresión revelala manera en que los sentimientos son culturalmente conceptualizados por los quechuahablantes del dialecto ayacuchano. Específicamente, el amor se entiende como si fuera un objeto y, por tanto, como una propiedad de ellos: su unidad. Es así como el pensamiento quechua permite estructurar la consumación de una relación sentimental como una propiedad posible de los objetos: su separación. Esta concepción resulta -en la cultura quechua- por el uso del verbo raki -y 'separar'.Separarse de una persona es acabar la relación con esta. Así, la metáfora conceptual EL AMOR ES UNA UNIDAD representa el matiz cultural referido a las relaciones sentimentales concebidas por el quechuahablante.

\subsubsection{Localidad en las entidades inmateriales}

El conjunto de expresiones que exponemos a continuación muestran el modo en que las entidades inmateriales adquieren existencia física. Esta adquisición es posible en tanto la localidad suponga la materialización de las entidades inmateriales.

\section{(A) LOS EVENTOS SON LUGARES}

Manachayqa yaykusunchik atipanakuyman.

Sig.Lit.: «Entonces entremos a competencia».

Sig. Fig.: Entonces hagamos la competencia.

En el contexto cultural andino, los concursos o las rivalidades -con el propósito de lograr algún fin- son materializados si se entienden como una locación. En este sentido, el concepto atipanakuy 'competencia' se comprende culturalmente como un lugar al que se aspira llegar. En el discurso quechua es posible que el quechuahablante marque el inicio del concurso. El sistema 
quechua permite semantizar este concepto, en primer lugar, a través del verbo yaykuy 'entrar' y, en segundo lugar, por medio del sufijo direccional -man 'hacia, a', lo cual evidencia que la meta del trayecto es un lugar. Presentamos a continuación el esquema de la referencia:

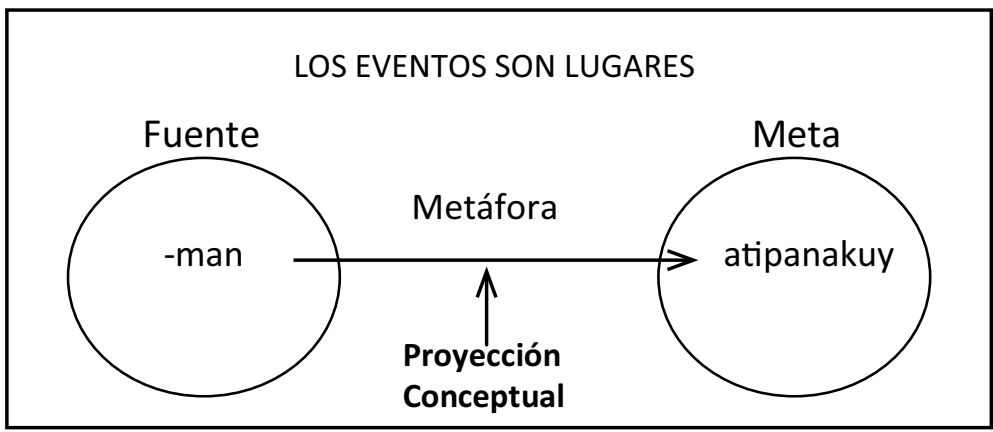

Fig. 5. Localidad en las entidades inmateriales

\section{(B) LAS EMOCIONES SON LUGARES}

Nuqataqa kay pinqakuymanmi churawanki.

Sig. Lit. : "A mí hacia esta verguienza me pones».

Sig. Fig. : Me pones en esta situación vergonzosa.

El ejemplo presenta un escenario en el que las emociones son concebidas culturalmente como lugares. La vergüenza se conceptualiza como un lugar en el cual el quechuahablante expresa la situación con respecto a tal estado. Se comprueba esta manera de concebir las emociones en la cosmovisión andina cuando se emplea el verbo chura -y 'poner' y el sufijo direccional -man 'hacia, a', con lo cual el estado referido se reinterpreta en el discurso quechua como una locación.

\subsubsection{Dimensionalidad en las entidades inmateriales}

El conjunto de expresiones que mostramos a continuación deja evidencia que las entidades inmateriales son reanalizadas como materiales en tanto pongan de manifiesto cierto grado de dimensionalidad.

(A) EL TIEMPO CRONOLÓGICO ES DIMENSIONAL

Hatunñam kanki mayllapipas llamkayñayá.

Sig. Lit. : "Grande ya eres, trabaja en algo». 
Sig. Fig. : Ponte a trabajar, ya eres adulto.

La expresión pone de manifiesto la manera en que el tiempo cronológico está siendo conceptualizado por el quechuahablante. El modo singular en que la cultura andina expresa el tiempo cronológico es través de un concepto dimensional. En el empleo del adjetivo hatunñan 'grande' se observa que la edad se entiende a partir de la dimensionalidad. Presentamos a continuación el esquema:

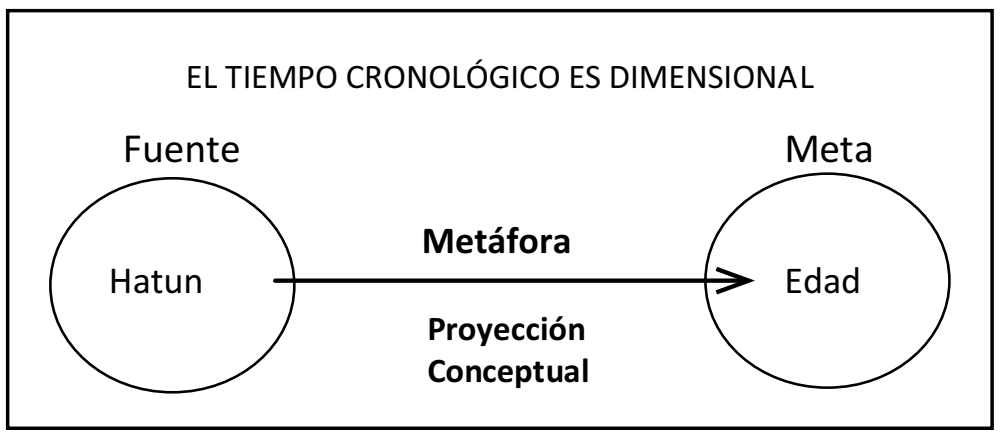

Fig. 6. Objetivización en las entidades inmateriales

\section{(B) LAS EMOCIONES SON DIMENSIONALIDADES}

\section{kananqa hatunllañam llakiy}

Sig. Lit. : «Ahora ya es grande mi pena».

Sig. Fig. : Ahora me siento muy triste.

En este caso, el concepto de llaki 'pena' se comprende como si fuera una entidad material pasible de atribuírsele dimensión. Esta atribución se verbaliza en el sistema quechua por medio del uso del adjetivo hatun 'grande'; por ello la interpretación del estado de ánimo -en el que la tristeza es profunda- se patentiza en el quechuahablante.

\section{Conclusiones}

Las metáforas ontológicas en el quechua ayacuchano se manifiestan a través de procesos conceptuales diversos, es decir, por medio demecanismos como la personificación y la cosificación. Estos procesos suponen la internalización de conceptos imaginísticos con los que el quechuahablante confiere ciertos atributos a las entidades carentes de vitalidad y existencia física en la cosmovisión andina, con lo cual se patentiza un trazo invariable entre lengua 
y cultura quechua. Este trazo demuestra la manera en que la complejidad se simplifica en el habla y pensamiento quechua.

Las metáforas ontológicas analizadas en el discurso quechua evidencian una serie de características propias de la cosmovisión andina. En lo concerniente al proceso de personificación, ellas cristalizan una relación directa y compacta entre las entidades carentes de vitalidad y animicidad. Las metáforas que están referidas al proceso de cosificación permiten constatar en el quechua(en tanto sistema y cultura) tres maneras en que la materialización es posible: objetivización, localidad y dimensionalidad.

Finalmente, las expresiones metafóricas quechuas de naturaleza ontológica constituyen una parte de la evidencia empírica para sostener el modo en que el quechuahablante expresa y concibe su entorno inmediato. Es decir, estas expresiones permiten interpretar la realidad en la que intervienen mecanismos cognitivos variados. Dichas expresiones forman parte del acervo cultural de los hablantes quechuas y están presentes permanentemente en su discurso cotidiano.

\section{Referencias bibliográficas}

CALVO, Julio (1993). Pragmática y gramática del quechua cuzqueño. Cusco: CBC.

CUENCA, M. y Joseph Hilferty (1999). Introducción a la lingüística cognitiva. Barcelona: Editorial Ariel S.A.

LAKOFF, George y Mark Johnson1988 [1980]. Metáforas de la vida cotidiana. Madrid: Ediciones Cátedra.

PARKER, Gary (1965). Gramática del quechua de Ayacucho. Lima: Universidad Nacional Mayor de San Marcos.

SOTO, Clodoaldo (2012). Diccionario funcional quechua - castellano -inglés. Perú: Lluvia Editores.

TORERO, Alfredo (1964). El quechua y la historia social andina. Lima: Universidad Ricardo Palma.

TORERO, Alfredo (2001.) Idiomas de los Andes. Lingüística e Historia. Lima: Editorial Horizonte e IFEA. 